\title{
THE BLASIUS EQUATION WITH THREE-POINT BOUNDARY CONDITIONS*
}

\author{
BY \\ L. G. NAPOLITANO \\ Polytechnic Institute of Brooklyn
}

\begin{abstract}
The Blasius equation subject to three-point boundary conditions, describing the interaction between two parallel streams, is solved by way of a series in terms of ascending powers of the ratio $\lambda=\left(u_{1}-u_{2}\right) / u_{1}$, where the $u_{i}^{\prime}$ 's are the outer streams' velocities.

The first three terms of the series are analytically expressed in terms of the repeated integrals of the complementary error function $\left(i^{n}\right.$ erfc $\left.\eta\right)$ and of the repeated integrals of the square of the successive integrals of the complementary error function $\left(j^{m} i^{n}\right.$ erfe $\left.\eta\right)$. These functions often appear in problems leading to extended heat-conduction type of equations. A recurrence formula for $j^{m} i^{n}$ erfc $\eta$ is established and formulae relating the functions $i^{n}$ erfc $(-\eta)$ and $j^{m} j^{n}$ erfc $( \pm \eta)$ to available tabulated values of the functions $i^{n} \operatorname{erfc}(\eta)$ are derived.

The first three approximations to the Blasius function and to its first two derivatives are also presented in tabulated form with four significant figures. Test on the convergence of the series has been made by comparison with some exact solutions obtained by high speed computing machine. The comparison, extended to the physically essentlal quantities, shows that:

(1) The Blasius function itself is slightly less accurate than its second and first derivatives.

(2) Two terms of the series for $\lambda$ up to 0.5 and three terms for $\lambda$ up to 0.7 yield extremely accurate results. The errors in the first two derivatives of the Blasius functions are always contained within less than one per cent.
\end{abstract}

1. Introduction. The solution of the Blasius equation with three-point boundary conditions has per se a considerable academic interest. The availability of closed form solution has, however, become a practical necessity in view of the recent findings which have shown the essential and unique role played by this equation in isobaric mixing flows. It can indeed be said that all the types of plane two-dimensional interactions between two streams are governed by the Blasius equation with three-point boundary conditions.

The reducibility of the basic Prandtl equations to the Blasius equation with pertinent boundary conditions was first shown by Görtler [1] ${ }^{* *}$ for the laminar incompressible mixing of uniform streams and, subsequently [2], for the turbulent case also. The present author showed [3] that the same happens for the compressible laminar case. A recent investigation by the present author [4] has also brought forth some evidence of an empirical correlation existing between turbulent and laminar compressible mixing. It was found that, under the assumption of a unitary turbulent Prandtl number, the velocity profiles are considerably independent of Mach numbers and density ratio and

\footnotetext{
*Received December 19, 1957. This work was sponsored in part by the NACA under Contract NAw 6480. Some of the results have appeared in Ref. [9] which was issued in a limited number of copies not for circulation.

**Numbers in brackets refer to the bibliography at the end of the paper.
} 
can therefore be deduced from the solution of the Blasius equation. This statement has an even larger implication insofar as it applies whenever the density ratio can be given a parabolic dependence on the velocity ratio. Thus the field of application of the Blasius function is widened to include a large variety of interactions between streams of different gases [5]. Solutions of the Blasius equation are furthermore needed in problems of laminar and turbulent mixing of non-uniform constant vorticity streams. These problems are solved by means of a series solution in terms of the "vorticity numbers": the zeroth order terms is the Blasius function and the coefficient of the equations for the higher order terms are all functions of the Blasius function and its derivatives [6].

The present paper is mainly concerned with the solution of the Blasius equation and not with its derivation for which reference is made to the pertinent literature. The solution is obtained as a series in terms of the parameter $\lambda=\left(u_{1}-u_{2}\right) / u_{1}$. The first three terms (up to $\lambda^{8}$ ) are given in explicit closed form and in tabulated forms. Owing to the complicated nature of the terms of the series, its convergence could not be formally established. The results of the present method are, however, compared with some exact solutions obtained by high speed computing machine calculations.

In the course of the mathematical treatment there often appeared successive repeated integrals of the complementary error function (symbolically indicated by $i^{n}$ erfc $\eta$ ) and successive repeated integrals of the square of the functions $i^{n}$ erfc $\eta$. As this feature is common to a large variety of physical problems which can be reduced to extended heat conduotion type of equations, a summary study of those functions is presented in Appendixes A and B. Formulae necessary to compute the values of $i^{n}$ erfc $(-\eta)$ in terms of the already tabulated values (up to $n=11$ ) of $i^{n}$ erfc $\eta$ are developed. A recurrence formula is established for the functions $j^{m} i^{n}$ erfc $\eta$ defined as the successive integrals of the functions $\left(i^{n} \text { erfe } \eta\right)^{2}$. Finally relationships giving the functions $j^{m} i^{*}$ erfe $\eta$ in terms of the functions $i^{n}$ erfc $\eta$ are derived which afford a rapid evaluation of the functions themselves.

This work is part of a program of investigation on mixing phenomena carried out at the Polytechnic Institute of Brooklyn under the supervision of Prof. Antonio Ferri.

2. Solution of the Blasius equation. The equation to be solved is

$$
f^{\prime \prime \prime}+2 f f^{\prime \prime}=0
$$

and it is subject to the following three-point boundary conditions

$$
\lim _{\zeta \rightarrow+\infty} f^{\prime}=1 \quad \lim _{\zeta \rightarrow-\infty} f^{\prime}=1-\lambda \quad f(0)=0 \quad 0 \leq \lambda \leq 1 .
$$

Quantities related to the mixing of two streams are expressible in terms of the function $f(\zeta)$ and its derivatives as follows

$$
\begin{aligned}
& \psi=2\left(\nu u_{1} x\right)^{1 / 2} f(\zeta), \\
& \zeta=\frac{1}{2} y\left(\frac{\nu x}{u_{1}}\right)^{-1 / 2}+k \\
& u=u_{1} f^{\prime}(\zeta) \\
& v=\left(\frac{u_{1} \nu}{x}\right)^{1 / 2}\left[(\zeta-k) f^{\prime}-f\right] .
\end{aligned}
$$


In these equations $x$ and $y$ are space coordinates whose origin is taken to be at the point where the interaction begins, $u$ and $v$ are the corresponding velocity components, $\psi$ is the stream function defined by $u=\psi_{x} ; v=-\psi_{0}$ and $\nu$ is the kinematic viscosity coefficient. The quantity $k$ is an arbitrary constant whose presence follows from an interesting property of the Prandtl boundary layer equations. These equations are invariant under the transformation

$$
\begin{aligned}
y_{1} & =y+s(x), \\
x_{1} & =x, \\
u(x, y) & =u\left(x_{1}, y_{1}\right), \\
v(x, y) & =v_{1}\left(x_{1}, y_{1}\right)-u\left(x_{1}, y_{1}\right) \frac{d s}{d x} .
\end{aligned}
$$

Asymptotic boundary conditions on the $x$-component of the velocity remain also unchanged while boundary conditions on the $y$-component are changed. The mathematical implication of this fact lies in the freedom of choosing arbitrarily the third boundary condition for the Blasius equation. The physical implication is the resulting indeterminacy of the wake orientation insofar as the transformation back into the physical plane cannot be performed unless $k$ is known. Additional physical considerations, such as the one suggested by von Kármán that a free wake be acted upon by a zero resultant force in the $y$-direction, will uniquely determine this constant $k$ and thus will fix the orientation of the wake. The solution herein presented relates to a wake whose streamline through the origin satisfies the equation $y=-2 k\left(\nu x / u_{1}\right)^{1 / 2}$.

The following series solution of Eq. (1) in ascending powers of $\lambda$ is sought

$$
f=\sum_{i} \lambda^{i} f_{i} .
$$

Equation (5) is substituted into Eq. (1) and the coefficients of the successive powers of $\lambda$ are set equal to zero. The zeroth order approximation must satisfy the following equation

$$
f_{0}^{\prime \prime \prime}+2 f_{0} f_{0}^{\prime \prime}=0
$$

with

$$
\begin{aligned}
& \lim _{\zeta \rightarrow+\infty} f_{0}^{\prime}=1, \\
& f_{0}(0)=0 .
\end{aligned}
$$

The pertinent solution is $f_{0}=\zeta$ and it corresponds, physically, to zero mixing. The equation for the first approximation is, by taking the zeroth order solution into account

$$
f_{1}^{\prime \prime \prime}+2 \zeta f_{1}^{\prime \prime}=0
$$

with

$$
\begin{gathered}
\lim _{\zeta \rightarrow+\infty} f_{1}^{\prime}=0, \quad \lim _{\zeta \rightarrow-\infty} f_{1}^{\prime}=-1, \\
f_{1}(0)=0 .
\end{gathered}
$$


The solution of Eq. (8) is

$$
f_{1}=(\pi)^{-1 / 2} \int_{0}^{\zeta} d t \int_{0}^{t} e^{-b \cdot} d b-\frac{1}{2} \zeta .
$$

Finally, the $i$ th approximation $(i \geq 2)$ must satisfy the equation

$$
f_{i}^{\prime \prime \prime}+2 \zeta f_{i}^{\prime \prime}=f_{1}^{\prime \prime} R_{i}(\zeta),
$$

where the $R_{i}(\zeta)$ are functions, at most, of the $(i-1)$ th solution and are given by

$$
R_{i}(\zeta)=-2 \sum_{h=0}^{i-1} f_{h+1} f_{i-h}^{\prime \prime} / f_{1}^{\prime \prime} .
$$

Equation (11) is subject to the boundary conditions

$$
\lim _{b \rightarrow+\infty} f_{i}^{\prime}(0)=0, \quad f_{i}(0)=0, \quad(i \geq 2)
$$

and admits the solution

$$
f_{i}=(\pi)^{1 / 2} C_{1, i} f_{1}-(\pi)^{-1 / 2} \int_{0}^{5} d t \int_{0}^{\infty} e^{-b \cdot} d b \int_{0}^{b} R_{i}(a) d a
$$

with

$$
C_{1, i}=-(\pi)^{-1} \int_{-\infty}^{\infty} e^{-b^{2}} d b \int_{0}^{b} R_{i}(a) d a .
$$

Successive approximations to the solution $f$ can thus be readily determined to any order.

The task is considerably simplified if Eq. (14) is expressed in terms of the complementary error function and of related functions. In this problem, indeed, as well as in several other problems leading to extended heat-conduction type of equation, the solution can be expressed rather simply in terms of the following functions

$$
\begin{aligned}
i^{n} \operatorname{erfc} \eta & =\int_{\eta}^{\infty} \int_{\eta_{2}}^{\infty} \cdots \int_{\eta_{n}}^{\infty}\left(\operatorname{erfc} \eta_{n}\right) d \eta_{2} \cdots d \eta_{n}, \\
j^{m} i^{n} \operatorname{erfc} \eta & =\int_{\eta}^{\infty} \int_{\eta_{2}}^{\infty} \cdots \int_{\eta_{m}}^{\infty}\left(i^{n} \operatorname{erfc} \eta_{n}\right)^{2} d \eta_{2} \cdots d \eta_{m}, \\
T_{m n}(\eta) & =\int_{\eta}^{\infty}\left(i^{m} \operatorname{erfc} t\right) \cdot\left(i^{n} \operatorname{erfc} t\right) d t .
\end{aligned}
$$

Of these functions only the first ones, usually referred to as repeated integrals of the complementary error function, have been studied. Hartree [7] has shown some of their properties and applications and, more recently, Kaye [8] has tabulated them, up to the eleventh repeated integral, for positive value of the argument.

The functions $i^{n}$ erfc $(-\eta)$ are considered in Appendix A wherein their expressions in terms of the functions $i^{n}$ erfe $\eta$ and their asymptotic behavior are presented. A summary study of the functions $j^{m} i^{n}$ erfc $\eta$ is given in Appendix B. Therein the existence of a recurrence formula is proved and expressions relating the functions $j^{m} i^{n}$ erfc $( \pm \eta)$ and $T_{n n}( \pm \eta)$ to the repeated integrals of the error function are given.

If simplified notation such as

$$
\begin{aligned}
i^{n} \operatorname{erfc} \eta & \equiv i^{n}, \\
j^{m} i^{n} \operatorname{erfc} \eta & \equiv j^{m} i^{n},
\end{aligned}
$$


are adopted, the first approximations to the function $f$ can be given simple analytical expressions as follows:

First approximation-

$$
\begin{aligned}
f_{1} & =\frac{1}{2}\left[i-(\pi)^{-1 / 2}\right], \\
f_{1}^{\prime} & =-\frac{1}{2} i^{0}=-\frac{1}{2} \operatorname{erfc} \zeta, \\
f_{1}^{\prime \prime} & =(\pi)^{-1 / 2} e^{-\zeta^{2}} .
\end{aligned}
$$

Second approximation-

$$
\begin{aligned}
f_{2} & =-(16 \pi)^{-1 / 2}\left[\frac{1}{2}-i^{0}\right]+i\left[\frac{i^{2}}{2}-\frac{1}{4}\right]+\frac{3}{4}\left[\frac{1}{3} \frac{(2)^{1 / 2}-1}{(\pi)^{1 / 2}}-j i\right], \\
f_{2}^{\prime} & =\frac{i^{0}}{4}-\frac{i^{-1}}{4(\pi)^{1 / 2}}-\frac{1}{2} i^{0} i^{2}+\frac{1}{4}(i)^{2} \\
f_{2}^{\prime \prime} & =(\pi)^{-1 / 2} e^{-\zeta^{2}}\left[(\pi)^{-1 / 2} \zeta-\frac{1}{2}+i^{2}\right] .
\end{aligned}
$$

TABLE 1

Blasius equation with three-point boundary conditions-Values of $f_{i}$

\begin{tabular}{ccccccr}
\hline \hline$\zeta$ & $f_{1}(\zeta)$ & $f_{1}(-\zeta)$ & $f_{2}(\zeta)$ & $f_{2}(-\zeta)$ & $f_{3}(\zeta)$ & $f_{3}(-\zeta)$ \\
0.00 & .0000 & .0000 & .0000 & .0000 & .0000 & .0000 \\
0.01 & -.0050 & .0050 & .0004 & -.0006 & .0002 & -.0002 \\
0.02 & -.0099 & .0101 & .0009 & -.0009 & .0004 & -.0004 \\
0.04 & -.0195 & .0204 & .0017 & -.0032 & .0009 & -.0009 \\
0.06 & -.0290 & .0310 & .0025 & -.0034 & .0013 & -.0013 \\
0.08 & -.0382 & .0418 & .0032 & -.0041 & .0017 & -.0018 \\
0.10 & -.0472 & .0528 & .0038 & -.0052 & .0021 & -.0023 \\
0.12 & -.0559 & .0640 & .0044 & -.0065 & .0025 & -.0028 \\
0.14 & -.0645 & .0755 & .0050 & -.0077 & .0029 & -.0033 \\
0.16 & -.0728 & .0872 & .0054 & -.0090 & .0033 & -.0038 \\
0.18 & -.0809 & .0991 & .0058 & -.0104 & .0037 & -.0043 \\
0.20 & -.0888 & .1112 & .0063 & -.0118 & .0041 & -.0048 \\
0.30 & -.1250 & .1750 & .0078 & -.0196 & .0058 & -.0075 \\
0.40 & -.1560 & .2440 & .0075 & -.0295 & .0074 & -.0104 \\
0.50 & -.1823 & .3177 & .0069 & -.0381 & .0085 & -.0136 \\
0.60 & -.2041 & .3959 & .0056 & -.0503 & .0098 & -.0171 \\
0.70 & -.2220 & .4779 & .0037 & -.0610 & .0107 & -.0209 \\
0.80 & -.2365 & .5635 & .0016 & -.0705 & .0113 & -.0250 \\
0.90 & -.2480 & .6520 & -.0005 & -.0778 & .0018 & -.0295 \\
1.00 & -.2570 & .7430 & -.0024 & -.0867 & .0120 & -.0342 \\
1.20 & -.2691 & .9309 & -.0060 & -.1017 & .0122 & -.0437 \\
1.40 & -.2758 & 1.1243 & -.0085 & -.1126 & .0121 & -.0529 \\
1.60 & -.2792 & 1.3208 & -.0102 & -.1199 & .0119 & -.0609 \\
1.80 & -.2809 & 1.5191 & -.0112 & -.1238 & .0117 & -.0674 \\
2.00 & -.2816 & 1.7184 & -.0117 & -.1268 & .0116 & -.0720 \\
2.20 & -.2819 & 1.9181 & -.0119 & -.1280 & .0115 & -.0750 \\
2.40 & -.2820 & 2.1189 & -.0120 & -.1286 & .0115 & -.0765 \\
2.60 & -.2821 & 2.3179 & -.0121 & -.1288 & .0115 & -.0771 \\
2.80 & -.2821 & 2.5179 & -.0121 & -.1289 & .0115 & -.0773 \\
3.00 & -.2821 & 2.7179 & -.0121 & -.1289 & .0115 & -.0773 \\
\hline & & & & & \\
\hline
\end{tabular}


Third approximation-

$$
\begin{aligned}
& f_{3}^{\prime}=F_{3}+F_{3}(-\infty) f_{1}^{\prime}, \\
& F_{3}=-\frac{i^{0}}{2}\left[\frac{1}{4}+\frac{\zeta^{2}-1}{2 \pi}+\left(i^{2}\right)^{2}+\frac{3}{2}\left(\frac{1}{4 \pi}-\frac{1}{16}-j^{2} i\right)\right] \\
& +\frac{1}{4}\left[2(\pi)^{-1 / 2} i^{2}-(i)^{2}\right]-\frac{i^{-1} i^{2}}{4(\pi)^{1 / 2}}+\frac{i^{-1}}{4(2 \pi)^{1 / 2}} \\
& -\frac{i^{2}}{2}\left[\frac{1}{\pi}-(i)^{2}-\frac{\zeta}{\pi}+\frac{3}{2} j i\right]+\frac{1}{4} \int_{\zeta}^{\infty}[i(t)]^{3} d t, \\
& f_{3}^{\prime \prime}=F_{3}(-\infty) f_{1}^{\prime \prime}+(\pi)^{-1 / 2} e^{-\zeta \cdot}\left[\frac{1}{4}+\frac{\zeta^{2}-1}{2 \pi}+\frac{(\pi)^{-1 / 2}}{2}\left(\frac{\zeta}{2}+i\right)\right. \\
& \left.-i^{2}\left(i^{2}-1\right)-(\pi)^{-1 / 2} i^{2} \zeta-\frac{3}{2} j^{2} i\right] \text {. }
\end{aligned}
$$

\begin{tabular}{|c|c|c|c|c|c|c|}
\hline$\zeta$ & $f_{1}^{\prime}(\zeta)$ & $f_{1}^{\prime}(-\zeta)$ & $f_{2}^{\prime}(\zeta)$ & $f_{2}^{\prime}(-\zeta)$ & $f_{z}^{\prime}(\zeta)$ & $f_{2}^{\prime}(-\zeta)$ \\
\hline 0.0 & -.5000 & -.5000 & .0454 & .0454 & .0222 & .0222 \\
\hline .01 & -.4943 & -.5056 & .0440 & .0468 & .0220 & .0224 \\
\hline .02 & -.4887 & -.5113 & .0426 & .0482 & .0218 & .0225 \\
\hline .04 & -.4774 & -.5225 & .0398 & .0510 & .0215 & .0229 \\
\hline .06 & -.4662 & -.5338 & .0370 & .0538 & .0211 & .0233 \\
\hline .08 & -.4550 & -.5450 & .0342 & .0566 & .0208 & .0236 \\
\hline .10 & -.4438 & -.5562 & .0314 & .0594 & .0204 & .0240 \\
\hline .12 & -.4326 & -.5674 & .0287 & .0621 & .0201 & .0244 \\
\hline .14 & -.4215 & -.5785 & .0260 & .0648 & .0197 & .0247 \\
\hline .16 & -.4105 & -.5895 & .0234 & .0674 & .0193 & .0251 \\
\hline .18 & -.3995 & -.6005 & .0218 & .0700 & .0190 & .0255 \\
\hline .20 & -.3886 & -.6113 & .0183 & .0725 & .0186 & .0259 \\
\hline .30 & -.3357 & -.6643 & .0080 & .0839 & .0166 & .0280 \\
\hline .40 & -.2858 & -.7142 & -.0033 & .0929 & .0146 & .0304 \\
\hline .50 & -.2397 & -.7602 & -.0109 & .0990 & .0123 & .0332 \\
\hline .60 & -.1981 & -.8019 & -.0163 & .1018 & .0098 & .0364 \\
\hline .70 & -.1611 & -.8389 & -.0195 & .1014 & .0075 & .0399 \\
\hline .80 & -.1289 & -.8710 & -.0210 & .0980 & .0052 & .0433 \\
\hline .90 & -.1015 & -.8984 & -.0209 & .0920 & .0034 & .0450 \\
\hline 1.00 & -.0786 & -.9213 & -.0197 & .0841 & .0018 & .0475 \\
\hline .20 & -.0448 & -.9551 & -.0154 & .0648 & .0000 & .0476 \\
\hline .40 & -.0238 & -.9761 & -.0105 & .0451 & -.0010 & .0439 \\
\hline .60 & -.0118 & -0.9882 & -.0064 & .0285 & -.0012 & .0369 \\
\hline .80 & -.0054 & -0.9945 & -.0035 & .0123 & -.0008 & .0278 \\
\hline 2.00 & -.0023 & -0.9977 & -.0017 & .0086 & -.0004 & .0010 \\
\hline 2.20 & -.0009 & -0.9991 & -.0008 & .0041 & -.0002 & .0107 \\
\hline 2.40 & -.0003 & -0.9996 & -.0003 & .0018 & -.0001 & .0045 \\
\hline 2.60 & -.0001 & -0.9999 & -.0001 & .0007 & -.0001 & .0018 \\
\hline 2.80 & .0000 & -1.0000 & .0000 & .0003 & .0000 & .0002 \\
\hline 3.00 & .0000 & -1.0000 & .0000 & .0001 & .0000 & .0000 \\
\hline
\end{tabular}

TABLE 2

Blasius equation with three-point boundary conditions-Values of $f_{i}^{\prime}$ 
TABLE 3

Blasius equation with three-point boundary conditions-Values of $f_{i}^{\prime \prime}$

\begin{tabular}{|c|c|c|c|c|c|c|}
\hline$\zeta$ & $f_{1}^{\prime \prime}(\zeta)$ & $f_{1}^{\prime \prime}(-\zeta)$ & $f_{2}^{\prime \prime}(\zeta)$ & $f_{2}^{\prime \prime}(-\zeta)$ & $f_{3}^{\prime \prime}(\zeta)$ & $f_{3}^{\prime \prime}(-\zeta)$ \\
\hline .00 & .5642 & .5642 & -.1410 & -.1410 & -.0176 & -.0176 \\
\hline .01 & .5641 & .5641 & -.1410 & -.1410 & -.0176 & -.0176 \\
\hline .02 & .5640 & .5640 & -.1409 & -.1409 & -.0177 & -.0177 \\
\hline .04 & .5633 & .5633 & -.1404 & -.1404 & -.0177 & -.0177 \\
\hline .06 & .5622 & .5622 & -.1395 & -.1395 & -.0178 & -.0178 \\
\hline .08 & .5606 & .5606 & -.1384 & -.1383 & -.0179 & -.0179 \\
\hline .10 & .5586 & .5586 & -.1370 & -.1367 & -.0180 & -.0180 \\
\hline .12 & .5561 & .5561 & -.1352 & -.1348 & -.0180 & -.0182 \\
\hline .14 & .5532 & .5532 & -.1332 & -.1326 & $-: 0182$ & -.0184 \\
\hline .16 & .5499 & .5499 & -.1309 & -.1300 & -.0186 & -.0188 \\
\hline .18 & .5462 & .5462 & -.1283 & -.1271 & -.0188 & -.0193 \\
\hline .20 & .5421 & .5421 & -.1255 & -.1239 & -.0190 & -.0200 \\
\hline .30 & .5156 & .5156 & -.1083 & -.1031 & -.0201 & -.0225 \\
\hline .40 & .4808 & .4808 & -.0874 & -.0760 & -.0216 & -.0255 \\
\hline .50 & .4394 & .4394 & -.0650 & -.0448 & -.0235 & -.0300 \\
\hline .60 & .3936 & .3936 & -.0430 & -.0121 & -.0265 & -.0345 \\
\hline .70 & .3456 & .3456 & -.0230 & .0195 & -.0250 & -.0350 \\
\hline .80 & .2975 & .2975 & -.0061 & .0478 & -.0218 & -.0320 \\
\hline .90 & .2510 & .2510 & .0070 & .0708 & -.0171 & -.0205 \\
\hline 1.00 & .2075 & .2075 & .0165 & .0875 & .0118 & -.0100 \\
\hline 1.20 & .1337 & .1337 & .0246 & .1011 & .0072 & .0100 \\
\hline 1.40 & .0795 & .0795 & .0233 & .0927 & .0031 & .0260 \\
\hline 1.60 & .0436 & .0436 & .0176 & .0722 & .0015 & .0435 \\
\hline 1.80 & .0221 & .0221 & .0114 & .0491 & .0028 & .0480 \\
\hline 2.00 & .0103 & .0103 & .0065 & .0297 & .0018 & .0440 \\
\hline 2.20 & .0045 & .0045 & .0033 & .0160 & .0007 & .0350 \\
\hline 2.40 & .0018 & .0018 & .0015 & .0078 & .0004 & .0220 \\
\hline 2.60 & .0006 & .0006 & .0006 & .0035 & .0002 & .0105 \\
\hline 2.80 & .0002 & .0002 & .0002 & .0014 & .0001 & .0050 \\
\hline 3.00 & .0001 & .0001 & .0001 & .0005 & .0001 & .0008 \\
\hline
\end{tabular}

The first three approximations to the functions $f(\zeta), f^{\prime}(\zeta)$ and $f^{\prime \prime}(\zeta)$ have been computed to six significant figures. Values of $f_{i}(\zeta), f_{i}^{\prime}(\zeta)$ and $f_{i}^{\prime \prime}(\zeta),(i=1,2,3)$ are tabulated in Tables 1 and 3 with four significant figures.

3. Accuracy of the solution. The convergence of the series given by Eq. (5) could not be formally established, owing to the complexity of the relative terms. Practical indications about the rapidity of the convergence, however, are derived by comparing the results with those of some exact solutions obtained with the D12 Differential Analyzer presently in operation at the Centro di Calcolo Elettronico of the University of Naples. ${ }^{1}$

The following indicative values for $\lambda$ were considered: $\lambda=0.2678 ; \lambda=0.4796$; $\lambda=0.5541 ; \lambda=0.6915$. Comparison was extended to the following physically meaningful quantities:

i) $f^{\prime}(0)$, (proportional to the component $u$ of the velocity along the $\xi$-axis);

1These calculations are part of a larger program of high speed machine solution of turbulent mixing flows sponsored by the United States Air Force through the Air Force Office of Scientific Research, Air Research and Development Command, under Contract AF 18(600)-693, Project No. 17500. The cooperation of Prof. Giorgio Savastano, Associate Director of the C. C. E., is gratefully acknowledged. 
ii) $f^{\prime \prime}(0)$, (proportional to the shear stress along the $x$-axis);

iii) $\lim _{\zeta \rightarrow \infty}\left[\zeta f^{\prime}-f\right]$, (proportional, for $k=0$, to the $y$-component of the velocity at the edges of the wake).

Values obtained from the exact solutions and from the first, second and third approximations to the function $f(\zeta)$ are listed in Table 4.

TABLE 4

Comparison between exact and approximate solutions

\begin{tabular}{lllll}
\hline & Exact & I Approx. & II Approx. & III Approx. \\
$\lambda=0.26783$ & 0.8698 & 0.8661 & 0.8693 & 0.8697 \\
$f^{\prime}(0)$ & 0.1406 & 0.1511 & 0.1410 & 0.1407 \\
$f^{\prime \prime}(0)$ & 0.0762 & 0.0755 & 0.0764 & 0.0762 \\
$\lim _{\zeta+\infty}\left[f^{\prime}-f\right]$ & 0.0755 & 0.0847 & 0.0862 \\
$\lim _{\zeta-\infty}\left[f^{\prime}-f\right]$ & 0.0865 & & & \\
$\lambda=0.47961$ & & 0.7602 & 0.7706 & 0.7730 \\
$f^{\prime}(0)$ & 0.7739 & 0.2706 & 0.2382 & 0.2362 \\
$f^{\prime \prime}(0)$ & 0.2360 & 0.1353 & 0.1381 & 0.1368 \\
$\lim _{\zeta+\infty}\left[f^{\prime}-f\right]$ & 0.1362 & 0.1353 & 0.1649 & 0.1734 \\
$\lim _{\zeta+\infty}\left[\zeta f^{\prime}-f\right]$ & 0.1765 & & & \\
$\lambda=0.55412$ & & 0.7229 & 0.7368 & 0.7406 \\
$f^{\prime}(0)$ & 0.7421 & 0.3126 & 0.2693 & 0.2663 \\
$f^{\prime \prime}(0)$ & 0.2660 & 0.1563 & 0.1600 & 0.1580 \\
$\lim _{\zeta+\infty}\left[\zeta f^{\prime}-f\right]$ & 0.1568 & 0.1563 & 0.1959 & 0.2090 \\
$\lim _{\zeta+\infty}\left[\zeta f^{\prime}-f\right]$ & 0.2154 & & & \\
$\lambda=0.69147$ & & 0.6543 & 0.6760 & 0.6833 \\
$f^{\prime}(0)$ & 0.6870 & 0.3901 & 0.3227 & 0.3169 \\
$f^{\prime \prime}(0)$ & 0.3158 & 0.1951 & 0.2009 & 0.1971 \\
$\lim _{\zeta+\infty}\left[\zeta f^{\prime}-f\right]$ & 0.1937 & 0.1951 & 0.2567 & 0.2822 \\
$\lim _{\zeta-\infty}\left[f^{\prime}-f\right]$ & 0.3003 & & & \\
\hline
\end{tabular}

The following comments are proper:

(1) The best agreement is obtained for $f^{\prime \prime}$, followed, in order, by $f^{\prime}$ and $f$.

(2) The accuracy decreases with $\lambda$ and, for a given $\lambda$, is greater for $\zeta$ large than for $|-\zeta|$ large.

(3) The $y$-component of the velocity at the lower edge of the wake always exhibits the maximum percental error.

(4) The first two approximations are more than satisfactory up to values of $\lambda=0.5$. The errors in $f^{\prime}(0)$ and $f^{\prime \prime}(0)$ are less than one per cent.

(5) Three terms of the series are needed for values of $\lambda$ greater than 0.5 . With three: terms the errors are nearly always contained within less than one per cent up to $\lambda$ equal to 0.7 . The only exception lies again in the value of the $y$-component of the velocity at the lower edge of the wake. The approximate value is $6 \%$ smaller than the exact one. 


\section{REFERENCES}

1. H. Görtler, Berechnung von Aufgaben der freien Turbulenz auf Grund eines neuen Naherung Sansatzes, Zamm Bd. 22, Nt. 5, 244-254 (Oct. 1942)

2. S. I. Pai, Fluid dynamic of jets, D. Van Nostrand Co., Inc., New York, 1954

3. L. G. Napolitano, Similar solutions in compressible laminar free mixing problems, Readers' Forum, J. Aeronaut. Sci. 23, No. 4 (April 1956)

4. L. G. Napolitano, Two-dimensional plane mixing of homogeneous and non-homogeneous streams, PIBAL Rept. No. 400, Nov. 1957

5. L. G. Napolitano, Turbulent mixing of two non-reacting gases, PIBAL Rept. No. 323, Jan. 1957

6. L. G. Napolitano, Incompressible mixing of a shear flow with fluid at rest, PIBAL Ropt. No. 318, Nov. 1957

7. D. R. Hartree, Some properties and applications of the repeated integrals of the error function, Mem. and Proc., Manchester Lit. and Phil. Soc. 80, No. 9, 85-102 (1935)

8. J. Kaye, $A$ table of the first eleven repeated integrals of the error function, J. Math. and Phys. (2) 34. 119-125 (1955)

9. L. G. Napolitano, The Blasius equation with three-point boundary conditions, PIBAL Rept. No. 319, Jan. 1957

\section{APPENDIX A}

The error function and its repeated integrals. The error function is defined by

$$
\operatorname{erf} \eta=\frac{2}{(\pi)^{1 / 2}} \int_{0}^{\eta} e^{-t^{2}} d t
$$

and the complement of the error function, erfc $\eta$, by

$$
\operatorname{erfc} \eta=1-\operatorname{erf} \eta=\frac{2}{(\pi)^{1 / 2}} \int_{\eta}^{\infty} e^{-t^{2}} d t
$$

The $n$th repeated integral of erfc $\eta$ is symbolically defined as

$$
i^{n} \operatorname{erfc} \eta=\int_{\eta}^{\infty}\left(i^{n-1} \operatorname{erfc} t\right) d t \quad(n \geq 1)
$$

with

$$
i^{0} \operatorname{erfc} \eta=\operatorname{erfc} \eta \text {. }
$$

The functions given by Eq. (A3) are tabulated, up to $n=11$, in Ref. [8] for positive values of the variable $\eta$.

It is of interest to extend the definition of $\imath^{n}$ erfc $\eta$ to negative values of $\eta$ as follows

$$
i^{n} \operatorname{erfc}(-\eta)=\int_{-\eta}^{\infty}\left(i^{n-1} \operatorname{erfc} t\right) d t
$$

and to see whether it is possible to express them as functions of the known tabulated values of $i^{n}$ erfe $\eta$.

In consistence with the notation given in Eq. (A3), the first derivative of the error function is defined by

$$
-\frac{d}{d \eta}(\operatorname{erfc} \eta)=i^{-1} \operatorname{erfc} \eta=\frac{2}{(\pi)^{1 / 2}} e^{-\eta^{2}} .
$$

It is, apparently, an even function, so that

$$
i^{-1} \operatorname{erfc}(-\eta)=i^{-1} \operatorname{erfc} \eta,
$$


whereas

$$
i^{0} \operatorname{erfc}(-\eta)=2-i^{0} \operatorname{erfc} \eta \text {. }
$$

By repeated application of the following recurrence formula [7], valid for $n \geq 1$

$$
2 n i^{n} \operatorname{erfc} \eta=i^{n-2} \operatorname{erfc} \eta-2 \eta i^{n-1} \text { erfc } \eta
$$

it is then easily verified that the following general identity holds

$$
i^{n} \operatorname{erfc}(-\eta)=(-1)^{n+1} i^{n} \operatorname{erfc} \eta+2 \sum_{n=0}^{n}\left[\frac{1+(-1)^{n-k}}{2}\right] \frac{1}{h !} \eta^{n} i^{n-n} \operatorname{erfc}(0) .
$$

In deriving Eq. (A10) it has been taken into account that Eq. (A9) yields, for $\eta=0$

$$
2 n i^{n} \operatorname{erfc}(0)=i^{n-2} \operatorname{erfc}(0)
$$

or

$$
i^{i} \operatorname{erfc}(0)=\frac{1}{2^{n}\left(\frac{1}{2} n\right) !} .
$$

Equation (A10) gives the required relation between the repeated integrals of the error function for negative and positive values of the independent variables. Thus, for instance, the first three integrals in the negative range of $\eta$ are simply expressed by

$$
\begin{aligned}
i \operatorname{erfc}(-\eta) & =2 \eta+i \operatorname{erfc} \eta \\
i^{2} \operatorname{erfc}(-\eta) & =-i^{2} \operatorname{erfc} \eta+\eta^{2}+2 i^{2} \operatorname{erfc}(0), \\
i^{3} \operatorname{erfc}(-\eta) & =i^{3} \operatorname{erfc} \eta+2 \eta i^{2} \operatorname{erfc}(0)+\eta^{3} / 3
\end{aligned}
$$

Apparently these repeated integrals will not converge as $\eta \rightarrow-\infty$. Their asymptotic behavior, for $|-\eta|$ large, is

$$
i^{n} \operatorname{erfc}(-\eta) \sim(-1)^{n+1} \frac{2}{(\pi)^{1 / 2}} \frac{e^{-\eta^{2}}}{(2 \eta)^{n+1}}+2 \sum_{h=0}^{n}\left[\frac{1+(-1)^{n-h}}{2}\right] \frac{\eta^{h}}{h !} i^{n-h} \operatorname{erfc}(0) .
$$

\section{Appendix B}

Repeated integrals of the functions $\left(\imath^{n} \operatorname{erfc} \eta\right)^{2}$. Let the successive integrals of the functions $\left(i^{n} \text { erfc } \eta\right)^{2}$ be symbolically indicated by

$$
\begin{array}{ll}
j^{m} i^{n} \operatorname{erfc} \eta=\int_{\eta}^{\infty}\left(j^{m-1} i^{n} \operatorname{erfc} t\right) d t & (m \geq 0) \\
& (n \geq 0)
\end{array}
$$

with

$$
j^{0} i^{n} \operatorname{erfc} \eta=\left(i^{n} \operatorname{erfc} \eta\right)^{2} .
$$

It is desired to express these functions in terms of the repeated integrals of the error function.

The relationship is immediate for the two particular cases: $n=-1$ ( $m$ any positive integers) and $m=1(n \geq 0)$.

Indeed when $m=1$ and $n=-1$, it is by definition

$$
j i^{-1} \operatorname{erfc} \eta=\int_{\eta}^{\infty}\left[\frac{2}{(\pi)^{1 / 2}} e^{-t^{\prime}}\right]^{2} d t
$$


so that [see Eq. (A3)]

$$
j i^{-1} \operatorname{erfc} \eta=\left(\frac{2}{\pi}\right)^{1 / 2} \operatorname{erfc}\left[\eta(2)^{1 / 2}\right] .
$$

Repeated integrations easily yield the required relation between $j^{m} i^{-1}$ erfc $\eta$ and the repeated integrals of erfc $\eta$ as

$$
j^{m} i^{-1} \operatorname{erfc} \eta=\frac{[2]^{1-(m / 2)}}{(\pi)^{1 / 2}} i^{m-1} \operatorname{erfc}\left[\eta(2)^{1 / 2}\right]
$$

A corresponding expression, valid for $m=1$ and any $n \geq 0$ can be obtained by repeated integrations by parts. As it is easy to verify, the following identity will result

$$
\begin{aligned}
j i^{n} \operatorname{erfc} \eta=\sum_{h=1}^{n+1}( & -1)^{h+1} \frac{[2(n-h+1)] ! n ! 2^{h-1}}{(2 n+1) !(n-h+1) !}\left\{i^{n-h} \operatorname{erfc~} \eta \cdot i^{n-h+1} \operatorname{erfc} \eta\right. \\
& \left.-\eta\left(i^{n-h+1} \operatorname{erfc} \eta\right)^{2}\right\}+(-1)^{n+1}(\pi)^{-1 / 2} \frac{n ! 2^{1 / 2+n}}{(2 n+1) !} \operatorname{erfc}\left[(2)^{1 / 2} \eta\right]
\end{aligned}
$$

In deriving Eq. (B6), the following identity

$$
j^{-1} i^{n} \operatorname{erfc} \eta=-\frac{d}{d \eta}\left(i^{n} \operatorname{erfc} \eta\right)^{2}=2 i^{n} \operatorname{erfc} \eta \cdot i^{n-1} \operatorname{erfc} \eta
$$

which constitutes an obvious extension of the definition (B1) to the case $m=-1$, has been taken into account.

In the most general case use must be made of a recurrence formula. This formula can be derived by successive and repeated integration by parts of Eq. (B1). By taking Eq. (A9) into consideration one obtains

$$
(2 n+m) j^{m} i^{n} \operatorname{erfc} \eta=\frac{1}{2} j^{m-2} i^{n} \operatorname{erfc} \eta-\eta j^{m-1} i^{n} \operatorname{erfc} \eta-j^{m} i^{n-1} \operatorname{erfc} \eta
$$

valid for $m \geq 1$ and $n \geq 0$.

The recurrence formula (B8) together with Eqs. (B5) and (B6) afford a rapid computation of the functions $j^{m} i^{n}$ erfc $\eta$. Their extensions to negative values of the argument are readily accomplished by means of Eq. (A10).

The first few functions $j^{m} i^{n}$ erfc $\eta$, are explicited and their values at $\eta=0$ are given. A simplified notation such as

$$
\begin{gathered}
i^{n} \operatorname{erfc} \eta \equiv i^{n} \\
j^{m} i^{n} \operatorname{erfc} \eta=j^{m} i^{n}
\end{gathered}
$$

is adopted. Thus

$$
\begin{aligned}
j^{0} & =i^{0} i^{-1}-\eta\left(i^{0}\right)^{2}-\left(\frac{2}{\pi}\right)^{1 / 2} i^{0}\left[\eta(2)^{1 / 2}\right] \\
j^{2} i^{0} & =\frac{1}{4}\left(i^{0}\right)^{2}-\frac{1}{2} \eta j i^{0}-(2)^{-3 / 2} i\left[\eta(2)^{1 / 2}\right] \\
j i & =\frac{1}{3} i i^{0}-\frac{1}{3} \eta(i)^{2}-\frac{1}{3} j i^{0} \\
j^{2} i & =\frac{1}{8}(i)^{2}-\frac{1}{4} \eta j i-\frac{1}{4} j^{2} i^{0}
\end{aligned}
$$


and

$$
\begin{array}{ll}
j i^{0}(0)=(\pi)^{-1 / 2}\left[2-(2)^{1 / 2}\right], & 4 j^{2} i^{0}(0)=1-\frac{2}{\pi}, \\
3 j i(0)=(\pi)^{-1 / 2}\left[(2)^{1 / 2}-1\right], & 4 j^{2} i(0)=\frac{1}{\pi}-\frac{1}{4} .
\end{array}
$$

To conclude, the integral

$$
T_{m n}(\eta)=\int_{\eta}^{\infty} i^{m} \operatorname{erfc} t \cdot i^{n} \operatorname{erfc} t d t \quad(n>m)
$$

will be evaluated.

Repeated integrations by parts and consideration of Eq. (B6) yield

$$
T_{m n}(\eta)=\frac{(-1)^{n}}{2}\left[\left(i^{n-h} \operatorname{erfc} \eta\right)^{2}\right]+\sum_{\nu=1}^{h}(-1)^{n-1}\left[i^{m+\nu} \operatorname{erfc} \eta \cdot i^{n-\nu+1} \operatorname{erfc} \eta\right]
$$

when $n-m=2 h+1$, and

$$
T_{m n}(\eta)=(-1)^{h} j i^{(m+n) / 2} \operatorname{erfc} \eta+\sum_{\eta=1}^{h}(-1)^{n-1}\left[i^{m+\nu} \operatorname{erfc} \eta \cdot i^{n-\nu+1} \operatorname{erfc} \eta\right]
$$

when $n-m=2 h$. 